\title{
Strong near-field optical localization on an array of gold nanodisks
}

\author{
Lionel Aigouy, ${ }^{1, a)}$ P. Prieto, ${ }^{2}$ A. Vitrey, ${ }^{2}$ J. Anguita, ${ }^{2}$ A. Cebollada, ${ }^{2}$ M. U. González, ${ }^{2}$ \\ A. García-Martín, ${ }^{2}$ J. Labéguerie-Egéa, ${ }^{3}$ and M. Mortier ${ }^{3}$ \\ ${ }^{1}$ Laboratoire de Physique et d'Etude des Matériaux, UMR CNRS 8213, ESPCI, 10 rue Vauquelin, \\ Paris Cedex 5 75231, France \\ ${ }^{2} I M M$ - Instituto de Microelectronica de Madrid (CNM-CSIC), Isaac Newton 8, PTM, Tres Cantos, \\ Madrid E-28760, Spain \\ ${ }^{3}$ Laboratoire de Chimie de la Matière Condensée de Paris, UMR CNRS 7574, ENSCP, \\ Rue Pierre et Marie Curie, Paris Cedex 5 75231, France
}

(Received 11 May 2011; accepted 10 July 2011; published online 19 August 2011)

\begin{abstract}
By scanning near-field optical microscopy, we measured the localization of the electromagnetic field on an array of gold nanodisks illuminated in a transmission mode. We experimentally observed that the field is localized between the disks, with a pattern oriented along the incident polarization direction. We also observed that the electromagnetic field rapidly decays above the nanodisks, showing a strong vertical localization. The experimental results are in good agreement with numerical simulations performed by a finite difference time domain method. This study provides quantitative information about the local optical properties of closely-packed nanodisks that can be used for applications in biochemical sensors and nanolithography. (C) 2011 American Institute of Physics. [doi:10.1063/1.3624749]
\end{abstract}

\section{INTRODUCTION}

The optical properties of metallic nanostructures have been extensively studied in the past 20 years. ${ }^{1-4}$ Compared to dielectrics, metallic particles have large absorption and scattering cross sections, which can be further increased by the presence of surface plasmon resonances. At the local scale, this results in the presence of strong evanescent fields localized near the particles. This strong localization of light can be used for surface-enhanced Raman scattering and surface enhanced fluorescence at the single molecule level, ${ }^{5-8}$ but also for developing plasmon resonance sensors ${ }^{9-11}$ and for performing near-field nanolithography. ${ }^{12,13}$ For all these applications, the knowledge of the field distribution on the structures is important. For instance, in the case of biochemical sensors, the position, the spatial extension of the light, and its vertical decay can determine the volume on which the devices are sensitive. In the case of near-field lithography, these parameters will characterize the penetration of light in a photoresist and the lateral resolution.

An interesting device for the aforementioned applications is the array of metallic particles. ${ }^{14-16}$ Several designs have been proposed and studied, including circular, elliptical, square, or triangular dots or C-shaped nanostructures. For arrays, the absorption and the local field distribution depend both on the wavelength and on several geometrical parameters, such as the particle size and shape and their separation. To study their properties, many experiments have been performed by transmission spectroscopy. However, this technique does not give information about the field localization on the structures, and many questions are being raised. For instance, is the field localized above the particles or between them? How fast does the field decay above the par-

${ }^{\text {a)} E l e c t r o n i c ~ m a i l: ~ l i o n e l . a i g o u y @ e s p c i . f r . ~}$ ticles? How does it depend on the incident polarization? Although these questions can be answered by performing numerical simulations with finite difference time domain (FDTD), finite/boundary element, or Green Dyadic methods, there is a lack of experimental observations of such effects. In this work, our aim is to directly observe the electromagnetic field distribution using a scanning near-field optical microscope (SNOM). Recently, many SNOM experiments have been performed on isolated or coupled nanostructures, ${ }^{17-20}$ but only very few studies have been achieved on arrays. Salerno et $a .^{21}$ and Salomon et $a .^{22}$ observed the localization of light on an array of gold nanoparticles with a SNOM, but the structures were separated from each other by one micrometer, which makes them almost isolated from each other. Bakker et al. ${ }^{23}$ recently measured the transmission of light through an array of $\mathrm{Au} / \mathrm{SiO}_{2} / \mathrm{Au}$ nanostructures using a SNOM operating in the illumination mode. They observed an enhancement of light at some locations on the sample, which is attributed to a nanoantenna effect. Another experiment carried out by Diessel et al. ${ }^{24}$ on arrays of C-shaped nanostructures showed a strongly polarizationdependent near-field pattern. In the same spirit, we present here a near-field experimental study of a simple periodic structure, namely an array of gold nanodisks fabricated on a glass substrate. Our aim is to determine where the light is localized after transmission through the structure. Our experiments, performed both in contact and non-contact modes, are in good agreement with numerical simulations performed by FDTD method.

\section{EXPERIMENTAL DETAILS}

The sample was fabricated by electron-beam lithography. The geometrical parameters of the structure were determined from the scanning electron microscopy (SEM) image 
represented in Fig. 1(a). The disks are 286 nm-diameter, 50 $\mathrm{nm}$-high, and the period is $500 \mathrm{~nm}$. This structure was found to have a broad plasmon resonance peak in the near-infrared between 800 and $900 \mathrm{~nm}$. For the SNOM experiments, we used a fluorescent particle glued at the end of a sharp tungsten tip as a probe [an SEM image of an example of the tip is shown in Fig. 1(b)]. The particle is a $\mathrm{PbF}_{2}$ nanocrystal codoped with erbium and ytterbium ions. ${ }^{25}$ It acts as a nanodetector, which absorbs the local field and re-emits it by fluorescence at a different wavelength. For the experiments, it was excited in the near-infrared at $\lambda=975 \mathrm{~nm}$, just above the plasmon resonance of the nanodisk array. At this wavelength, the fluorescent ions efficiently absorb the local field and emit a strong fluorescence in the visible $(\lambda=550 \mathrm{~nm})$. This absorption process involves two photons (up-conversion mechanism ${ }^{26}$ ), and the fluorescence intensity varies as the square of the field excitation intensity. Since the particle contains several hundreds of fluorescent centers oriented in many directions, it is sensitive to all the components of the electromagnetic field. Therefore, by collecting the fluorescence intensity as a function of the tip position on the sample, we obtain an image which represents the square of the total field intensity on the surface.

The experimental configuration is schematically drawn in Fig. 1(c). ${ }^{27,28}$ The sample is illuminated in a transmission mode with a linearly polarized, intensity-modulated laser beam. The tip is set on a piezoelectric cube that enables displacements in the three directions on the sample. As illustrated in Fig. 1(d), experiments can be achieved by following the surface topography (constant distance mode) or in a noncontact mode (constant height mode). In the constant distance mode, the tip-sample distance is regulated using the tapping mode (oscillation amplitude $\cong 20 \mathrm{~nm}$, frequency $=6 \mathrm{KHz}$ ). In the constant height mode, the feedback loop is disabled and the tip flies freely above the surface. Scans in a

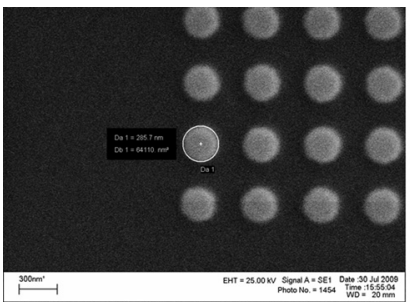

(a)

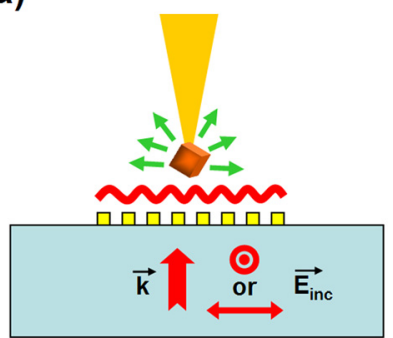

(c)

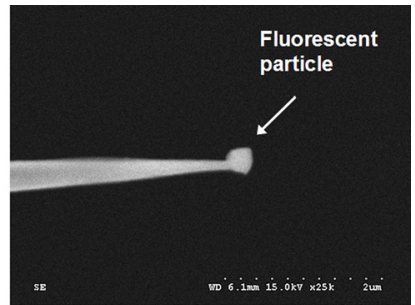

(b)
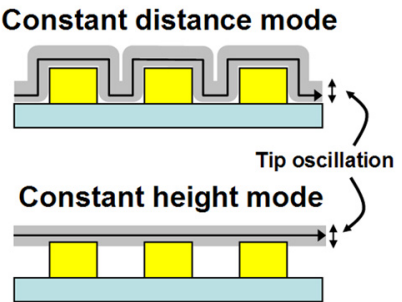

(d)

FIG. 1. (Color online) SEM picture of the gold nanodisks array (a), SEM picture of an example of the tip used in the experiment (b), sketch of the experimental setup (c), and description of the constant distance and constant height modes (d). plane perpendicular to the surface can also be performed to measure the vertical localization of the electromagnetic field.

\section{RESULTS AND DISCUSSION}

We show in Fig. 2 the topography and the near-field optical images measured on the nanodisks in the constant distance mode for two different incident polarization directions. The near-field pattern consists of periodic bright spots located between the disks. It appears that the near-field light and the disks are aligned in the direction of the incident polarization. When the polarization direction is rotated, the position of the bright spots also changes, which shows that they are not a tipinduced artifact. However, a quantitative analysis is difficult to perform in this scanning mode, because the tip follows the topography and penetrates between the disks.

To avoid this z-motion complication and to be able to compare the near-field images to theoretical simulations, we performed measurements in the constant height mode. For that, the tip is oscillated vertically as if it was going to operate in the tapping mode (the free space oscillation amplitude is set to $\cong 30 \mathrm{~nm}$ ), but the usual feedback loop is disabled. At the beginning of the scan, the tip is positioned so that it slightly touches the top of the disks [see Fig. 1(d)]. Then, during the scan, its oscillation amplitude is slightly attenuated when it encounters the surface of each disk and vibrates in free space when it is between two disks. To avoid any damage of the sample and the tip, the oscillation damping above the disks has to be very small (less than $20 \%$ of the free oscillation), so the scanning plane needs to be parallel to the sample surface and the scan has to be fast to minimize thermal drifts. $(\mathrm{nm})$
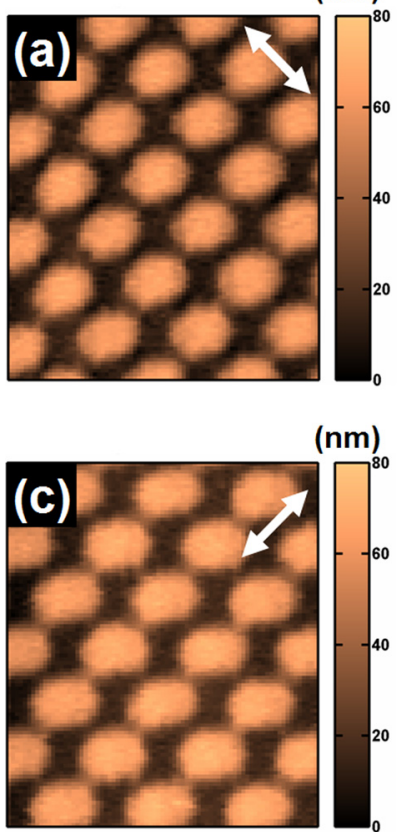

$1 \mu \mathrm{m}$
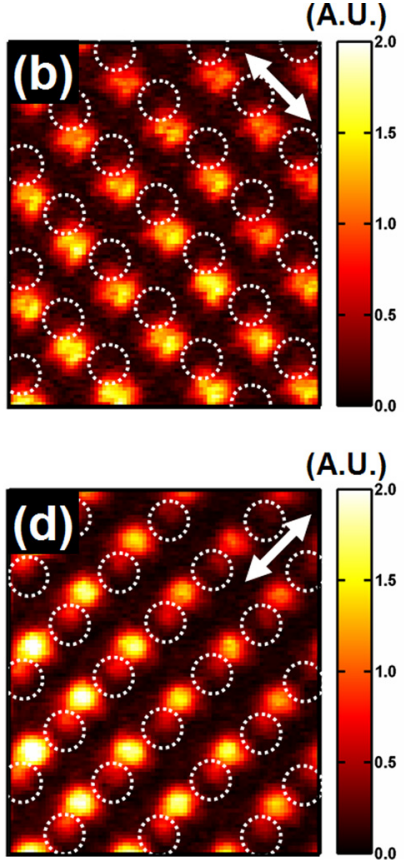

FIG. 2. (Color online) Topography $(\mathrm{a}, \mathrm{c})$ and corresponding "constant distance" experimental SNOM images $(b, d)$ on the nanodisks array for two incident polarization directions (indicated by the white arrows). 
We show in Fig. 3 the tip oscillation amplitude and the SNOM images measured with this scanning mode for two different incident polarization directions. The oscillation amplitude images [represented in Figs. 3(a) and 3(b)] show the top surface of the disks and allow us to situate their position with regard to the optical signal. The SNOM images [Figs. 3(c) and 3(d)] are different from the ones obtained in the constant distance mode shown in Fig. 2. In particular, the features have a more elongated shape and appear to be comprised of two lobes situated between the disks. As in the constant distance mode, when the incident polarization is rotated, the orientation of the pattern also rotates, which shows that the presence of the lobes is not a tip-induced artifact.

To check the correctness of the measured SNOM signal and to understand why the local field has such a distribution, we have simulated the near-field pattern using a finite difference time domain (FDTD) numerical method. Since the fluorescent particle is sensitive to all the electromagnetic field
(A.U.)

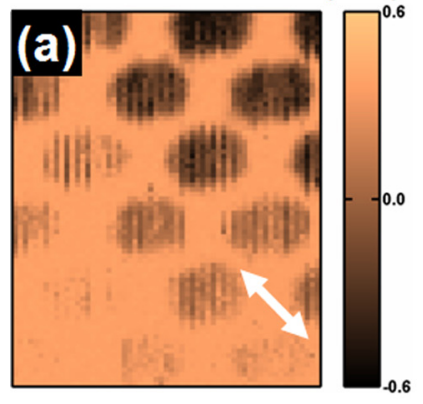

(A.U.)

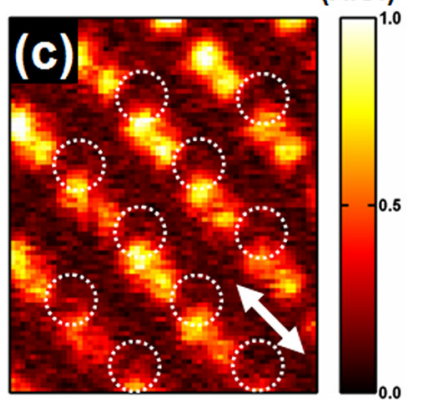

(A.U.)

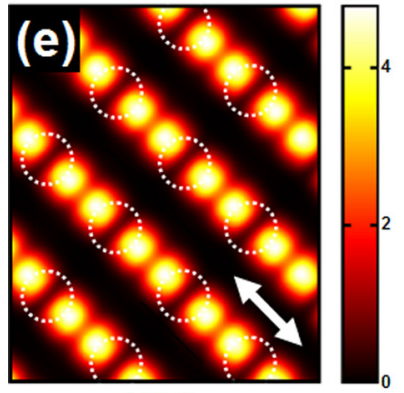

$500 \mathrm{~nm}$
(A.U.)

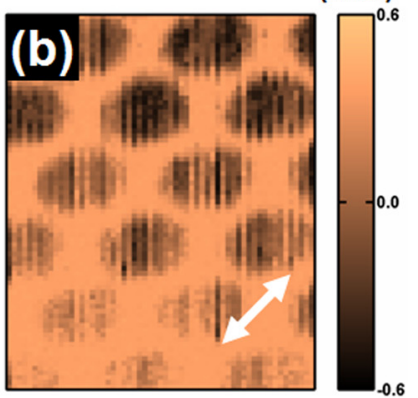

(A.U.)

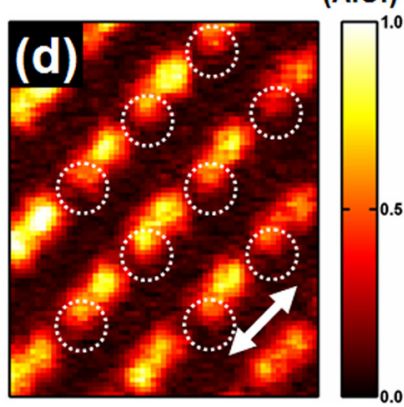

(A.U.)

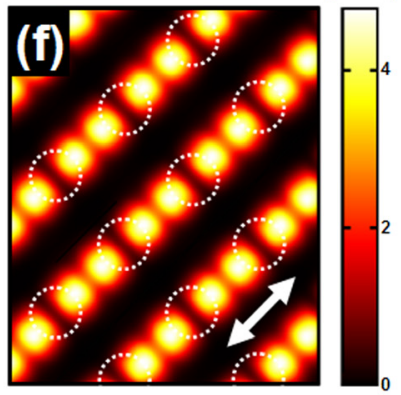

FIG. 3. (Color online) Tip oscillation amplitude or error signal (a,b), corresponding experimental constant height SNOM images measured at the nanodisks array for two different polarization directions (c,d), and numerical simulation of the SNOM signal (e,f). The simulation represents the square of the total field intensity in a plane parallel to the sample surface averaged on the fluorescent particle size $\left(160 \times 160 \times 160 \mathrm{~nm}^{3}\right)$. The incident polarization direction is indicated by the white arrows. components, and since the emitted fluorescence varies with the square of the excitation, we calculated the square of the total field intensity $\left\|\mathrm{E}_{\text {tot }}\right\|^{4}$. To take into account the fluorescent particle size, we integrated the signal over a volume $\mathrm{V} .^{29}$ The measured signal can then be represented by the quantity

$$
\mathrm{S}=\int_{\mathrm{V}}\left\|\mathrm{E}_{\mathrm{tot}}\right\|^{4} \mathrm{dV}
$$

The simulated near-field patterns are shown in Figs. 3(e) and 3(f). A good agreement is obtained between the simulations and the experiments when the particle is considered as a $160 \times 160 \times 160 \mathrm{~nm}^{3}$ large cube. Such dimensions correspond roughly to the size of the fluorescent particle we usually use for our experiments (between $100 \mathrm{~nm}$ and $300 \mathrm{~nm}$ ). As for the experimental SNOM image, the pattern is composed of two lobes located between the disks and whose relative orientation depends on the incident polarization direction. Note that the effect of averaging over a finite volume $\mathrm{V}$ only broadens the size of the lobes, but does not change the shape of the pattern.

A more quantitative comparison is shown in the higher resolution images of Fig. 4. The intensity of the simulated image has been normalized to allow the comparison with the experimental one. A good quantitative agreement is observed in terms of relative intensities. The cross-sections parallel to the incident polarization (cross-section A) exhibit a very similar shape and contrast. From cross-sections B, one can also see that the intensity of the light drops almost to zero between two disks in the direction perpendicular to the incident polarization. All the light is concentrated between the disks in the direction of the incident polarization. A similar local field distribution with two lobes has been observed indirectly on a polymer lithographically modified by the near-field of silver nanoparticles. ${ }^{30}$ Note that such a field

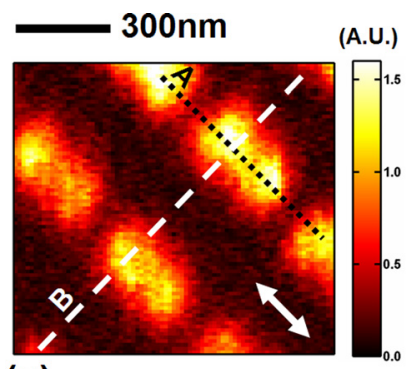

(a)

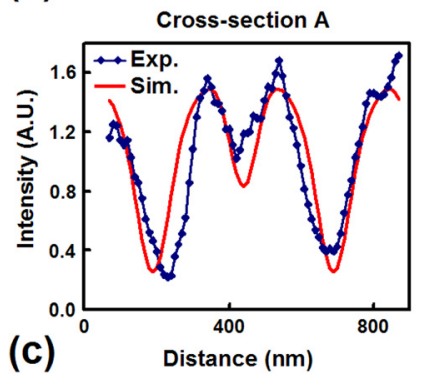

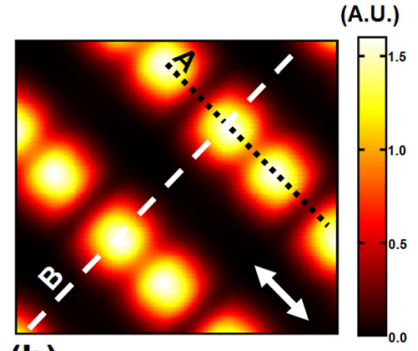

(b)

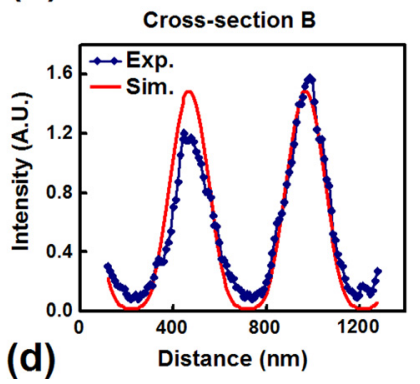

FIG. 4. (Color online) Experimental (a) and simulated (b) SNOM images of the array of nanodisks. The cross-sections parallel (direction A) and perpendicular (direction B) to the incident polarization are given in (c) and (d), respectively. The position of the gold disks is the same as the ones shown in Fig. 3. 


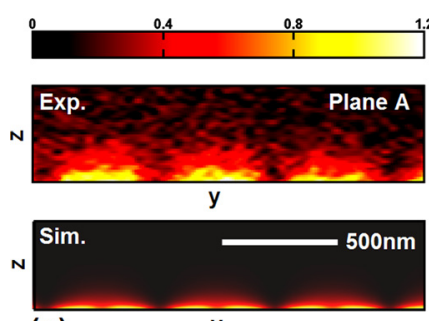

(a)
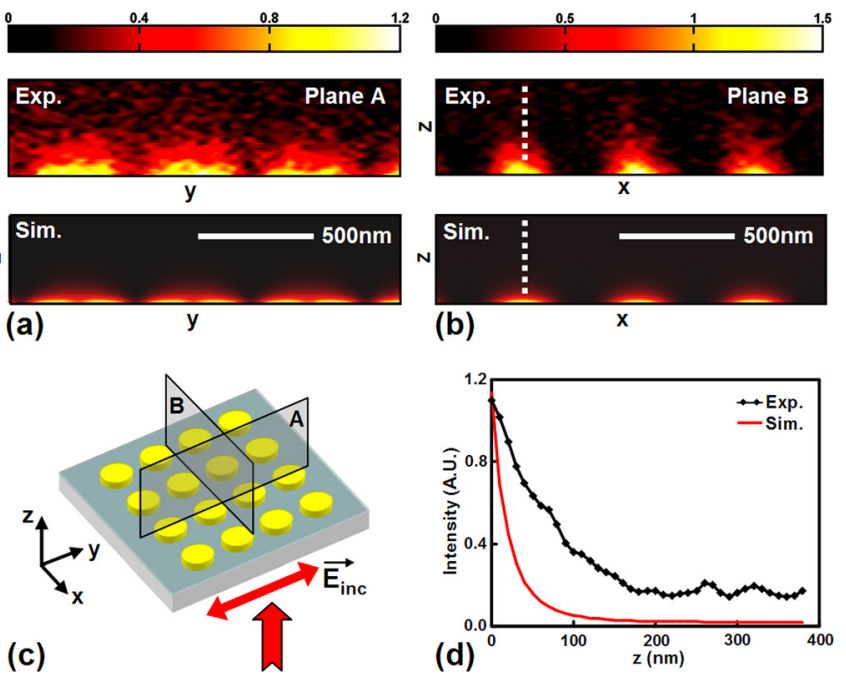

(b)

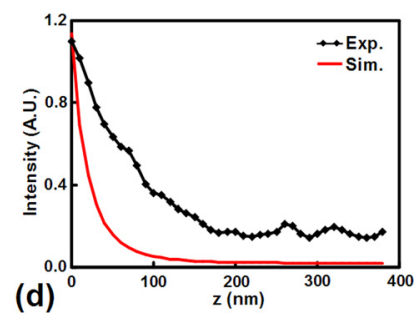

FIG. 5. (Color online) Experimental and simulated near-field images in a plane parallel (a) and perpendicular (b) to the incident polarization direction. The scanning planes are illustrated in (c). The experimental and simulated vertical decays extracted from the dotted line in (b) are shown in (d).

distribution with a two-lobe structure strongly depends on the periodicity of the array. For the sample studied here, the distance between adjacent disks is large (more than $200 \mathrm{~nm}$ ), so near-field interactions are avoided. The situation would be different if the disks were separated by only a few nanometers. In that case, near-field coupling effects between disks would induce a merging of the lobes and would lead to even stronger local field intensities. ${ }^{31-33}$

Another interesting parameter related to the structure is the vertical localization of the local field. In particular, if the aim is to develop chemical sensors or to perform nanolithography, one has to optimize how the light decays vertically on the structures. To infer this important parameter, we also performed scans in a plane perpendicular to the surface. Experimental and simulated images are shown in Fig. 5. The two-lobe structure still appears along the incident polarization direction. It is also worth noting that the intensity of the signal strongly diminishes when the tip gets far from the surface. The SNOM signal drops by a factor of 3 at $100 \mathrm{~nm}$ above the surface and is ten times smaller at $200 \mathrm{~nm}$. This vertical localization is confirmed by the simulated images that exhibit an even stronger decay. The difference between the experiments and the simulations may be due to the fact that the tip freely scans the space above the structure. Then, although its displacements are well-controlled, the tipsample separation is subject to thermal drifts that can modify the real relative distance, giving some uncertainty in the vertical direction. From these images, we can see that an important amount of light is confined at a short distance from the disks and that the transmitted far-field is very weak. In a near-field lithography experiment or for other surface patterning applications, modifications would therefore only occur at a very close distance to the surface.

\section{SUMMARY AND CONCLUSIONS}

With the help of a scanning near-field optical probe, we observed the localization of light on an array of gold nano- disks illuminated in a transmission mode and for $\lambda=975 \mathrm{~nm}$, slightly above the plasmon resonance peak. The near-field appears to be formed of a periodic two-lobe pattern located between the disks in the direction of the incident polarization. We also observed that the light intensity rapidly decays in the vertical direction above the structure, showing a strong nearfield localization. In contrast to experiments performed with fiber-type probes ${ }^{21-24}$ or scattering tips, ${ }^{19,20}$ our measurements directly represent the intensity of the total field located on the surface (or more precisely, the square of the total field intensity). They indicate, in three dimensions, the zones of the structure that would be active if we use it as a chemical sensor or for performing near-field nanolithography.

\section{ACKNOWLEDGMENTS}

This work was supported by the European Union (EU) project Nanomagma under contract NMP3-SL-2008-214107. We also acknowledge the Spanish MICINN for partial funding under "FUNCOAT" CONSOLIDER INGENIO 2010 Grant No. DCS2008-00023 and "MAGPLAS" Grant No. MAT2008-06765-C02-01/NAN. L.A. thanks Rémi Carminati for helpful discussions and Loïc Lalouat for his contribution to the development of the SNOM system.

${ }^{1}$ C. F. Bohren, and D. R. Huffman, Absorption and Scattering of Light by Small Particles (Wiley, New York, 1998).

${ }^{2}$ K. L. Kelly, E. Coronado, L. L. Zhao, and G. C. Schatz, J. Phys. Chem. B 107, 668 (2003).

${ }^{3}$ S. A. Maier and H. A. Atwater, J. Appl. Phys. 98, 011101 (2005).

${ }^{4}$ N. Fang, H. Lee, C. Sun, and X. Zhang, Science 308, 534 (2005).

${ }^{5}$ J. Zhang, Y. Fu, M. H. Chowdhury, and J. R. Lakowicz, Nano Lett. 7, 2101 (2007).

${ }^{6}$ A. Mohammadi, V. Sandoghdar, and M. Agio, New. J. Phys. 10, 105015 (2008).

${ }^{7}$ J. B. Khurgin and G. Sun, J. Opt. Soc. Am. B 26, 83 (2009).

${ }^{8}$ K. Kneipp, Y. Wang, H. Kneipp, L. T. Perelman, I. Itzkan, R. R. Dasari, and M. S. Feld, Phys. Rev. Lett. 78, 1667 (1997).

${ }^{9}$ A. D. McFarland and R. P. Van Duyne, Nano Lett. 3, 1057 (2003).

${ }^{10}$ G. Raschke, S. Kowarik, T. Franzl, C. Sönnichsen, T. A. Klar, J. Feldmann, A. Nichtl, and K. Kürzinger, Nano Lett. 3, 935 (2003).

${ }^{11}$ S. S. Aćimović, M. P. Kreuzer, M. U. González, and R. Quidant, ACS Nano 3, 1231 (2009).

${ }^{12}$ A. F. Koenderink, J. V. Hernández, F. Robicheaux, L. D. Noordam, and A. Polman, Nano Lett.7, 745 (2007).

${ }^{13}$ P. G. Kik, A. L. Martin, S. A. Maier, and H. A. Atwater, Proc. SPIE 4810, 7 (2002).

${ }^{14}$ R. P. Van Duyne, A. J. Haes, and A. D. McFarland, Proc. SPIE 5223, 197 (2003).

${ }^{15}$ S. Enoch, R. Quidant, and G. Badenes, Opt. Express 12, 3422 (2004).

${ }^{16}$ E. J. Smythe, E. Cubukcu, and F. Capasso, Opt. Express 15, 7439 (2007).

${ }^{17}$ J. Prikulis, H. Xu, L. Gunnarsson, M. Käll, and H. Olin, J. Appl. Phys. 92, $6211(2002)$

${ }^{18}$ H.-Y. Lin, C.-H. Huang, C.-H. Chang, Y.-C. Lan, and H.-C. Chui, Opt. Express 18, 165 (2010).

${ }^{19}$ R. Esteban, R. Vogelgesang, J. Dorfmüller, A. Dmitriev, C. Rockstuhl, C. Etrich, and K. Kern, Nano Lett. 8, 3155 (2008).

${ }^{20}$ M. Schnell, A. Garcia-Etwarri, A. J. Huber, K. Crozier, J. Aizpurua, and R. Hillenbrand, Nat. Photonics 3, 287 (2009).

${ }^{21}$ M. Salerno, N. Félidj, J. R. Krenn, A. Leitner, F. R. Aussenegg, and J. C. Weeber, Phys. Rev. B 63, 165422 (2001).

${ }^{22}$ L. Salomon, C. Charbonnier, F. de Fornel, P. M. Adam, P. Guérin, and F. Carcenac, Phys. Rev. B 62, 17072 (2000).

${ }^{23}$ R. M. Bakker, V. P. Drachev, H.-K. Yuan, and V. M. Shalaev, Opt. Express 12, 3701 (2004).

${ }^{24}$ D. Diessel, M. Decker, S. Linden, and M. Wegener, Opt. Lett. 35, 3661 (2010). 
${ }^{25}$ J. Labéguerie, G. Dantelle, P. Gredin, and M. Mortier, J. Alloys Compd. 451, 563 (2008).

${ }^{26}$ F. Auzel, Chem. Rev. 104, 139 (2004).

${ }^{27}$ L. Aigouy, Y. De Wilde, and M. Mortier, Appl. Phys. Lett. 83, 147 (2003).

${ }^{28}$ L. Aigouy, P. Lalanne, H. Liu, G. Julie, V. Mathet, and M. Mortier, Appl. Opt. 46, 8573 (2007).

${ }^{29}$ B. Wang, L. Aigouy, E. Bourhis, J. Gierak, J. P. Hugonin, and P. Lalanne, Appl. Phys. Lett. 94, 011114 (2009).
${ }^{30}$ C. Hubert, A. Rumyantseva, G. Lerondel, J. Grand, S. Kostcheev, L. Billot, A. Vial, R. Bachelot, P. Royer, S.-H. Chang, S. K. Gray, G. P. Wiederrecht, and G. C. Schatz, Nano Lett. 5, 615 (2005).

${ }^{31}$ K.-H. Su, Q.-H. Wei, X. Zhang, J. J. Mock, D. R. Smith, and S. Schultz, Nano Lett. 3, 1087 (2003).

${ }^{32}$ O. Lecarme, T. Pinedo-Rivera, K. Berton, J. Berthier, and D. Peyrade, Appl. Phys. Lett. 98, 083122 (2011).

${ }^{33}$ Y. Tanaka, H. Ishiguro, H. Fujiwara, Y. Yokota, K. Ueno, H. Misawa, and K. Sasaki, Opt. Express 19, 7726 (2011). 